\title{
The global star formation law: from dense cores to extreme starbursts
}

\author{
Yu Gao \\ Purple Mountain Observatory, 2 West Beijing Road, Nanjing 210008; and National Astronomy \\ Observatory, Chinese Academy of Sciences, Beijing, P.R. China \\ yugao@pmo.ac.cn,ygao@nrao.edu
}

\begin{abstract}
Active star formation (SF) is tightly related to the dense molecular gas in the giant molecular clouds' dense cores. Our HCN (measure of the dense molecular gas) survey in 65 galaxies (including 10 ultraluminous galaxies) reveals a tight linear correlation between $\mathrm{HCN}$ and IR (SF rate) luminosities, whereas the correlation between IR and CO (measure of the total molecular gas) luminosities is nonlinear. This suggests that the global SF rate depends more intimately upon the amount of dense molecular gas than the total molecular gas content. This linear relationship extends to both the dense cores in the Galaxy and the hyperluminous extreme starbursts at high-redshift. Therefore, the global SF law in dense gas appears to be linear all the way from dense cores to extreme starbursts, spanning over nine orders of magnitude in IR luminosity.
\end{abstract}

Keywords. stars: formation - ISM: molecules - infrared: galaxies - radio lines: galaxies galaxies: high-redshift

\section{Introduction}

Schmidt (1959) law of star formation (SF) was first formulated in terms of local SF rate $S F R$ proportional to the HI gas density with a power index n $\left(S F R \sim \rho^{n}, \mathrm{n} \sim 1-3\right)$ as the atomic gas was then known as the major component of interstellar gas reservoir to possibly form stars. More than a decade later, observations of the CO line emission in the Milky Way and external galaxies enabled by millimeter ( $\mathrm{mm}$ ) astronomy suggest that stars are forming in the giant molecular clouds (GMCs). From 80's, e.g., Kennicutt (1983) found little evidence in parameterizing the global SF law in external galaxies in terms of the total HI gas. But he succeeded in terms of the disk-averaged surface $S F R$ and total surface gas densities of both $\mathrm{HI}$ and $\mathrm{H}_{2}$ (Kennicutt 1989). However, a well determined power index $n$ was still not practical. Other researchers also obtained a wide range of the power index from 1 to 3. Recently, Kennicutt (1998) seemed to obtain a well determined slope of 1.4 though discrepancy exists (e.g., Heyer et al. 2004).

We here show that the SF law in terms of the dense gas is a rather simple linear relation (Gao \& Solomon 2004a, GS04a) and is straight-forward to understand in what we have learned so far from the physics of SF. The cool atomic gas has the potential to convert into molecular form to possibly provide the fuel to make stars, yet even the bulk of molecular gas and most regions of GMCs are not making stars except for those tiny dense cores. Great Observatories indeed begin to directly link/picture the heavily obscured dusty regions of active SF, embedded in GMCs as mapped by $\mathrm{mm} / \mathrm{sub}-\mathrm{mm}$ telescopes, to the current massive SF.

\section{Correlations among FIR, HCN \& $\mathrm{CO}$ in galaxies}

High-dipole moment molecules such as $\mathrm{HCN}$ trace more than an order of magnitude higher gas density than that of $\mathrm{CO}$. A major HCN survey in a wide range of 65 galaxies, 
including 10 ultraluminous infrared galaxies (ULIRGs), tripled the sample of galaxies with global HCN measurements (Gao \& Solomon 2004b). Analysis of the various relationships among the global HCN, CO, and FIR luminosities can be statistically conducted for the first time (GS04a).

The strong IR-HCN correlation is linear and extremely tight over 3 orders of magnitude in luminosity, when compared to the non-linear IR-CO correlation (GS04a). While the high luminosity of ULIRGs requires an elevated SF efficiency of the total molecular gas indicated by $L_{\mathrm{IR}} / L_{\mathrm{CO}}$, the $S F R$ per unit of dense molecular gas, the SF efficiency of the dense molecular gas indicated by $\left(L_{\mathrm{IR}} / L_{\mathrm{HCN}}\right)$ is almost constant and independent of the IR luminosity or total $S F R$. Further, GS04a find the surprising absence of any correlation between $L_{\mathrm{IR}} / L_{\mathrm{HCN}}$ and $L_{\mathrm{CO}} / L_{\mathrm{HCN}}$, yet a still strong correlation between $L_{\mathrm{IR}} / L_{\mathrm{CO}}$ and $L_{\mathrm{HCN}} / L_{\mathrm{CO}}$ (Fig. 1). This suggests that the $\mathrm{HCN}-\mathrm{IR}$ correlation is more physical than the CO-IR correlation and that the global SF efficiency depends on the fraction of the molecular gas in a dense phase $\left(L_{\mathrm{HCN}} / L_{\mathrm{CO}}\right)$. This is somehow reminiscent of the poor HI-IR correlation vs. the better CO-IR correlation discovered more than two decades ago.

The direct consequence of the linear IR-HCN correlation is that the SF law in terms of dense gas has a power law index of 1, which is different from the widely used Kennicutt (1998) law of a slope of 1.4 for the disk averaged $S F R$ as a function of the total gas (HI and $\mathrm{H}_{2}$ ). As we show in next section that this 1.4 law is not unique, neither valid for normal spiral galaxies nor for extreme starbursts/ULIRGs.
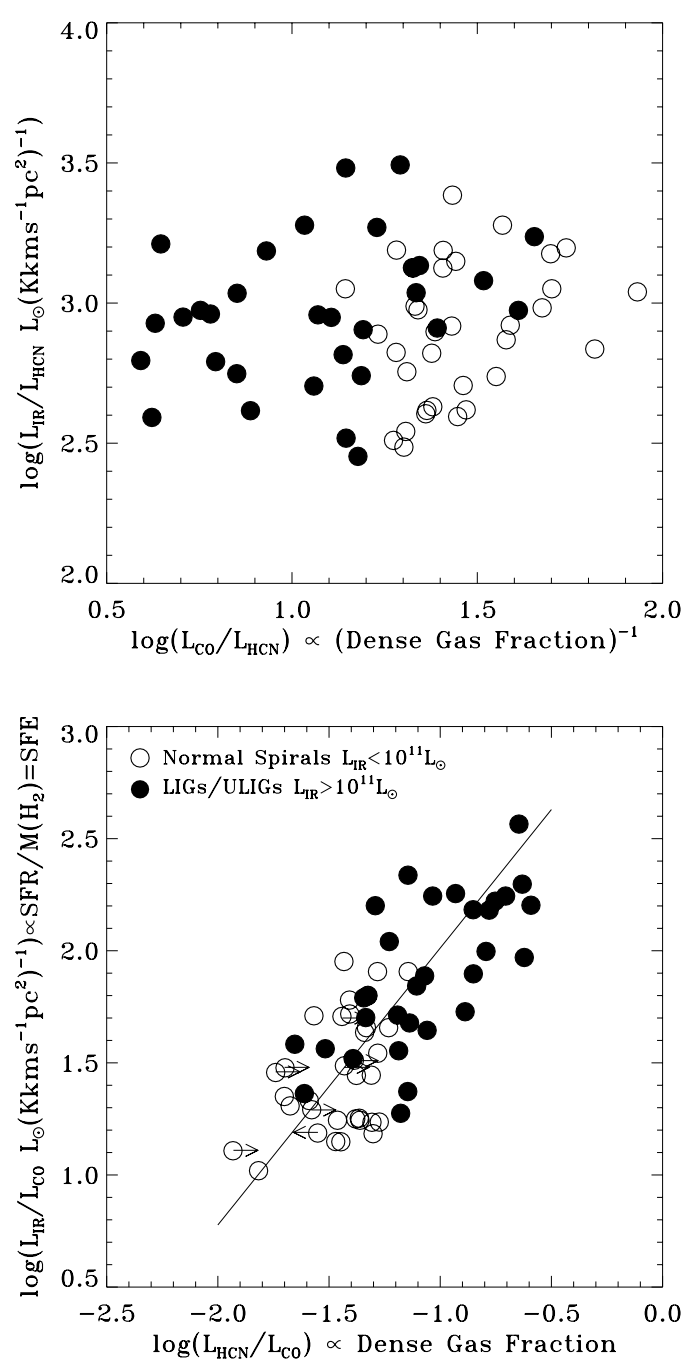

Figure 1. No correlation is observed between $L_{\mathrm{IR}} / L_{\mathrm{HCN}}$ and $L_{\mathrm{CO}} / L_{\mathrm{HCN}}$ (top), yet there is still strong correlation between $L_{\mathrm{HCN}} / L_{\mathrm{CO}}$ and $L_{\mathrm{IR}} / L_{\mathrm{CO}}$. The IR-CO correlation can simply be a result of the much better correlations of IR- $\mathrm{HCN}$ and $\mathrm{HCN}-\mathrm{CO}$.

\section{The global SF law in dense gas}

The $S F R-M_{\mathrm{H}_{2}}$ (IR-CO) is essentially linear up to $S F R \sim 20 \mathrm{M}_{\odot} / y r$ (Fig. 2a). This seems to also be true in terms of the mean surface densities of the $S F R$ and molecular gas mass for the nearest galaxies with spatially resolved observations (e.g., Wong \& Blitz 2002). Thus the linear form of the global SF law in terms of total molecular gas 
density as traced by $\mathrm{CO}$ for normal galaxies is due to the constant dense gas mass fraction ( $\mathrm{HCN} / \mathrm{CO})$ in normal galaxies (GS04a). For normal spirals, the SF law is linear in terms of both the total molecular gas and the dense molecular gas. A fit for the normal galaxies in the IR-CO correlation in $\mathrm{HCN}$ sample gives a slope of 1.0. But there is a poor correlation between the $S F R$ and the gas surface density in Kennicutt (1998) normal galaxy sample, difficult to derive a reasonable slope from normal galaxy sample alone.

A direct orthogonal regression fit for all galaxies in HCN sample leads to a slope of 1.4 (the least-squares fit slope is 1.3). These fits are almost identical to the SF law power index in Kennicutt's (1998) 36 circumnuclear starbursts sample. It is obvious from Fig. 2a that only galaxies with $S F R$ $>20 \mathrm{M}_{\odot} / \mathrm{yr}$ (mostly ULIRGs) lie above the slope 1 . The combination of normal galaxies and ULIRGs leads to a fit of 1.4. Therefore, this slope is not a universal slope at all as it changes according to the sample selection. The Kennicutt's (1998) 1.4 slope is determined mostly from the starburst sample. The circumnuclear starbursts have some of the characteristics of ULIRGs, e.g., a high dense gas fraction indicated by high $\mathrm{HCN} / \mathrm{CO}$ ratio.

When we add more ULIRGs into the sample, the slope becomes steeper, and the fits lead to a slope of 1.7. It is clear that the ULIRGs steepen the slope of the fit. There also appears to be a trend that some normal spirals with the lowest $\Sigma_{\mathrm{SFR}}$ and $\Sigma_{\mathrm{H}_{2}}$ in Kennicutt's sample tend to lie below the 1.4 fit. Adding more extreme galaxies, both ULIRGs and low luminosity galaxies, tends to steepen the slope towards 2. Therefore, it is difficult to derive a unique 1.4 power law based upon the total molecular gas or the total gas content.

The SF law in terms of dense gas has a unique slope of 1 (Fig. $2 \mathrm{~b}$ ) since
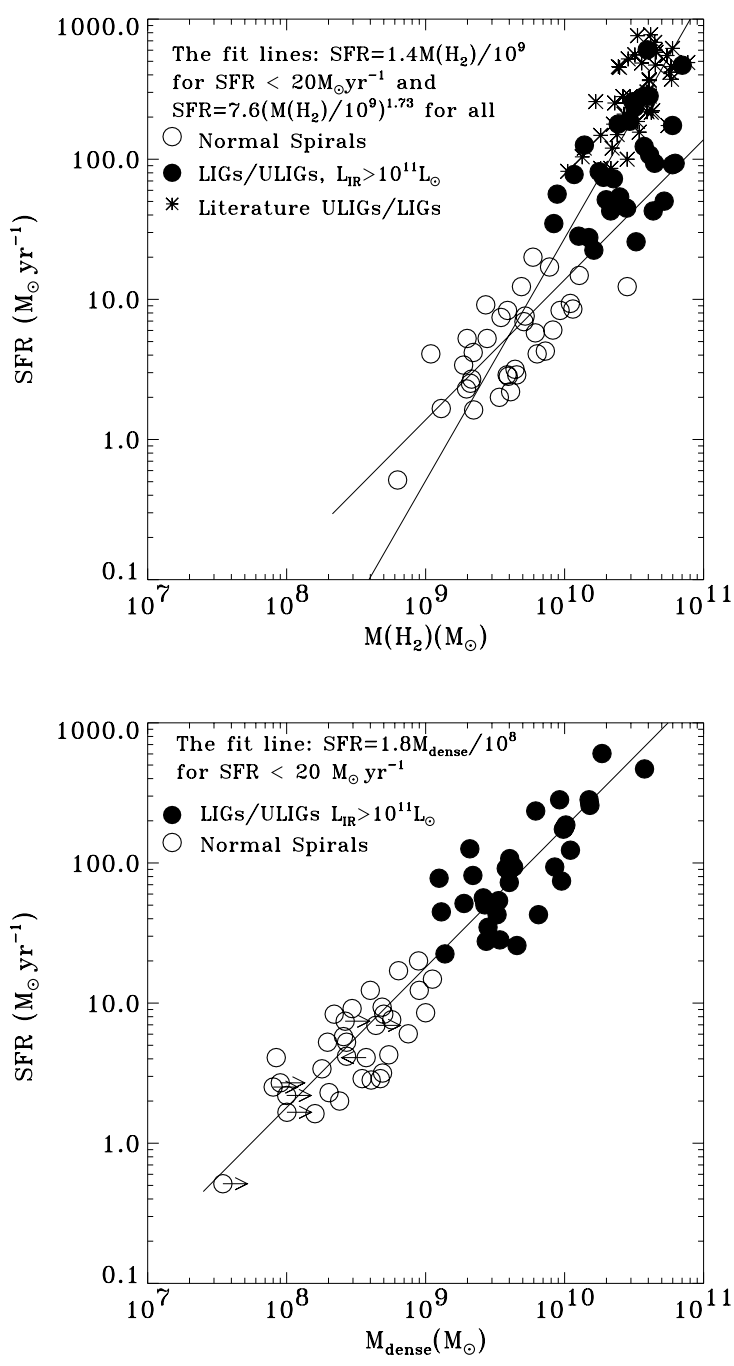

Figure 2. There is no unique SF law in terms of molecular gas or total gas (top) since the power index changes with the sample. But we do observe a linear SF law in dense gas for all star-forming galaxies.

the global $S F R$ is linearly proportional to the mass of the dense molecular gas. Parameterization in terms of observable mean surface densities of the dense molecular gas and the $S F R$ won't change the slope of 1 as both quantities are simply normalized by the same galaxy disk area. This linear SF law in dense gas seems valid for all star-forming galaxies. 
Remarkably, the $\mathrm{HCN}-\mathrm{IR}$ linear correlation is also valid to GMC dense cores implying the same physics drives the active SF in both dense cores and galaxies (Wu et al. 2005). New sensitive HCN line observations of four high-redshift submillimeter (sub-mm) galaxies and QSOs with the VLA including first possible HCN detection of a submm galaxy (Gao et al. 2006), combined with previous $\mathrm{HCN}$ detections and upper limits (e.g., Carilli et al. 2005) strongly suggest galaxies at high-redshift follow this same linear law (Fig. 3). The SF law in dense gas appears linear all the way from dense cores to extreme starbursts at high-z, spanning over nine orders of magnitude in $S F R$ or FIR luminosity.

The $S F R$ in a galaxy depends linearly on the dense molecular gas content as traced by $\mathrm{HCN}$, regardless of the galaxy luminosity or the presence of a "starburst", and not the total molecular gas and/or atomic gas traced by CO and/or HI observations respectively. Since dense molecular cloud cores are the sites of high mass SF, it is the physical properties, location and mass of these cores that set the $S F R$. A detailed SF law can be determined from observations directly probing the Milky Way cloud cores with spatially resolved measurements and the resolved measurements in nearby star-forming galaxies can bridge the dense cores with the galaxies. The global $S F R$ of a star-forming system is best characterized by its mass of dense gas, $S F R \sim \mathrm{M}\left(\right.$ dense $\left.\mathrm{H}_{2}\right)$. The gas density traced by $\mathrm{HCN}$ emission is apparently near the threshold for rapid $\mathrm{SF}$.

\section{Concluding remarks}

Although $S F R$ indicated FIR correlates with the various cold neutral gas reservoirs ( $\mathrm{HI}, \mathrm{H}_{2}, \mathrm{HCN}$-dense $\mathrm{H}_{2}$ ), the FIR-HI is the worst among all and the FIR-CO is non-linear and has larger scatter than that of FIR-HCN. The best and tightest FIR-HCN correlation is a linear relation which implies, under the assumption of the constant conversion factors, a linear SF law that $S F R$ is simply proportional to the amount of dense molecular gas available to form high mass stars. Other SF laws based on FIR-CO or FIR- $\left(\mathrm{HI}+\mathrm{H}_{2}\right)$ won't have a unique power index. This linear FIR-HCN relation appears to be valid all the way from dense cores in GMCs to extreme starbursts at high-z, revealing the same physics that massive SF drives most of the energy output in all these systems.

\section{Acknowledgements}

I wish to express my gratitudes to my collaborators, particularly Phil Solomon, for their contributions. China NSF (distinguished young scholars) \& Chinese Academy of Sciences (hundred-elite) are thanked for their supports. 


\section{References}

Carilli, C.L., Solomon., P.M., Vanden Bout, P.A., et al. 2005, ApJ 618, 586

Gao, Y. \& Solomon, P.M. 2004a, ApJ 609, 271 (GS04a)

Gao, Y. \& Solomon, P.M. 2004b, ApJS 152, 63

Gao, Y., Carilli, C.L., Solomon, P.M. \& Vanden Bout, P.A. 2006, ApJ submitted

Heyer, M.H., Corbelli, E., Schneider, S.E. \& Young, J.S. 2004, ApJ 602, 723

Kennicutt, R.C. 1983, ApJ 272, 54

Kennicutt, R.C. 1989, ApJ 344, 685

Kennicutt, R.C. 1998, ApJ 498, 541

Schmidt, M. 1959, ApJ 129, 243

Wong, T. \& Blitz, L. 2002, ApJ 269, 157

Wu, J., Evans, N.J.,II, Gao, Y., et al. 2005, ApJ 635, L173

\section{Discussion}

KenNicutT: The beautiful aspect of your result is that the dense cores do not seem to care about whether they reside in Orion or Arp 220 - they form stars with the same core efficiency. But doesn't this simply reword the problems of explaining why the efficiency of core formation scales with total gas density?

GaO: No. The dense cores do seem to form massive stars with the same efficiency regardless of where they reside. But the core formation and the efficiency of core formation depend upon the location and/or environment the putative cores reside. I'm not exactly sure how the efficiency of core formation scales with total gas density, but I'd think the efficiency of core formation scales with the fraction of gas at high density, rather than total gas or total molecular gas density. For example, a gas-rich low surface brightness galaxy could have nearly same disk-averaged total surface gas density as that of a starburst galaxy, but the starburst has high fraction of molecular gas, particularly dense molecular gas, therefore, a high star formation rate.

ELMEGREEN: I like to interpret these observations in a different way. It seems the extra power of 1.4 in the Kennicutt scaling relation is from the rate at which low density gas evolves toward high density gas. But for observations of only high-density gas, like yours, their evolution is not needed anymore and then the power of density is 1, not 1.4. The really important point of your observations seems to be that there is a universal critical density for star formation. Your observations are close to this density.

GAO: Yes, agree. It seems that a SF law in terms of low density gas needs extra power of 1.4 or higher. But the problem in low density gas (either $\mathrm{HI}$ or $\mathrm{H}_{2}$ or both) is that it is often difficult to derive a SF law with a unique power. As I showed that the SF law even in terms of the total molecular gas does not have a fixed 1.4 power and the situation is even worse in terms of atomic gas or total gas because of the poor correlation between $\mathrm{SF}$ rate and HI gas. On the other hand, a SF law in terms of total molecular gas (thus low density) for normal spirals does have a power index of 1 , same as that in terms of dense molecular gas probed by HCN observations. But the power index in terms of total molecular gas needs to be increased when extreme (ultraluminous and/or low surface brightness) galaxies are added/considered. 